\title{
Method Prediction for the Environment of Vibration Structure in Consideration with Elastic Joint
}

\author{
Yan Yang ${ }^{1}$ \\ ${ }^{1}$ School of Transportation and Automotive Engineering, Panzhihua University, Sichuan Panzhihua, 617000, China
}

\begin{abstract}
In order to accurately and efficiently predict the environment of vibration structures with elastic joints, the BUSH equivalent elastic joints unit of MSC. Nastran software was used to deduce the natural frequencies of structures with elastic joints by using the Fixed Interface Mode Synthesis Method (FIMSM).An example structure with elastic joint is designed, and both FIMSM and traditional FEM are used to calculate the first 30 order natural frequencies of the example structure. The results show that, compared with FEM, the relative calculation error of FIMSM is within $\pm 1.31 \%$. Consequently, via using BUSH element to simulate the elastic joint, FIMSM possesses the ability to predict the Environment of Vibration Structure considering elastic joint precisely and efficiently.
\end{abstract}

\section{Introduction}

The aircraft will experience complex and cruel vibration environment during launch and flight. For ensuring the high reliability of the aircraft, it must pass the ground vibration environment test. Formulating vibration environmental conditions reasonnably is crucial to the development of aircraft. Too low environmental conditions will lead to under-testing, making the aircraft unable to fully expose potential defects during ground tests, and the aircraft may fail to launch and fly in the actual environment.Excessive environmental conditions will lead to over-tests, requiring the aircraft to pass ground tests that are higher than the actual environment, which will increase the difficulty of aircraft development and lead to excessive development cycles and wasted funds ${ }^{[1,2]}$. When there is a large amount of measured environmental data, the environmental conditions formulated based on statistical induction methods are closest to the actual environment. However, for the newly-researched aircraft, that exist many uncertain parameters and the lack of measured environmental data, so the need to formulate the environmental conditions itself is often contradictory to the requirements of the aircraft development for environmental conditions. In this case, accurate and efficient prediction for the vibration environment is an inevitable choice to solve the problem $^{[3]}$.

However, the aircraft is a very complex structural system. There are often a large number of elastic connections (such as bolt connections) in complex structures. The impact of elastic connections on the vibration characteristics of the structure cannot be ignored $^{[4]}$. Therefore, a reasonable equivalent elastic connection is an important prerequisite for accurately predicting the structural vibration environment.
Literature [5,6] used the elastic connection in the equivalent structure of a six-degree-of-freedom scalar spring-damping system to conduct a preliminary study on the method of predicting the vibration environment of the structure considering the elastic connection, and obtained certain research results. The equivalent method does not take into account the coupling effect of the elastic connections, which is inconsistent with the actual engineering. Literature [7], through a large number of calculations and comparisons, combined with related finite Meta-theoretical knowledge, the stiffness matrix of the BUSH element was derived from the numerical results. It is found that the stiffness matrix of a single node of a BUSH element is not a diagonal matrix, and there are coupling stiffness terms for vertical displacement and rotation angle, which can be more accurately equivalent to the elastic connection in the actual engineering structure. In view of this, BUSH units have also been widely used in aerospace structural modeling. However, when the traditional FEM is used to predict the aircraft's vibration environment directly, if the prediction accuracy is to be improved, the structure of the aircraft must be finely modeled, which will lead to a sudden increase in calculation costs, so it is necessary to develop a more efficient method for predicting the structural vibration environment. Literature $[8,9]$ pointed out that FIMSM is an efficient method to deal with the dynamic characteristics of complex structures, so using FIMSM to accurately and efficiently predict the structural vibration environment of elastic connections has practical feasibility.

To sum up, this study uses the elastic connection in the equivalent complex structure of the BUSH unit in MSC. Nastran software. Based on the FIMSM, the natural frequency of the structure considering the elastic connection is theoretically derived, and the correctness

\footnotetext{
*Yang Yan: pzh_yangyan@126.com
} 
ofthe method is verified by numerical examples. The conclusions of this study have certain theoretical research and engineering application value.

\section{BUSH unit stiffness matrix}

Literature [7] based on the back-calculation of numerical results. First, the stiffness matrix of the BUSH unit was output using DMAP language. Then, the input parameters were changed and the stiffness matrix was changed. Finally, an analytical expression of the stiffness matrix $\mathrm{K}_{\mathrm{b}}$ of the BUSH unit was obtained by analyzing a large number of calculation results. The stiffness matrix $\mathrm{K}_{\mathrm{b}}$ is divided into 2 nodes, as shown below:

$$
K_{b}=\left[\begin{array}{ll}
K_{11} & K_{12} \\
K_{21} & K_{22}
\end{array}\right]
$$

In the above formula, the analytic expressions of the four partitioned matrix $\mathrm{K}_{11}, \mathrm{~K}_{12}, \mathrm{~K}_{21}$, and $\mathrm{K}_{22}$ are as follows:

$$
\begin{aligned}
& \boldsymbol{K}_{11}=\left[\begin{array}{cccccc}
k_{1} & & & & & \\
0 & k_{2} & & & \text { Symmetry } \\
0 & 0 & k_{3} & & \\
0 & 0 & 0 & k_{4} & & \\
0 & 0 & -\frac{k_{3} L}{2} & 0 & k_{5}+\frac{k_{3} L^{2}}{4} & \\
0 & \frac{k_{2} L}{2} & 0 & 0 & 0 & k_{6}+\frac{k_{2} L^{2}}{4}
\end{array}\right] \\
& \boldsymbol{K}_{12}=\boldsymbol{K}_{21}{ }^{\top}=\left[\begin{array}{cccccc}
-k_{1} & & & & \\
0 & -k_{2} & & & \text { Anti-Symmetry } \\
0 & 0 & -k_{3} & & & \\
0 & 0 & 0 & -k_{4} & & \\
0 & 0 & \frac{k_{3} L}{2} & 0 & -k_{5}+\frac{k_{3} L^{2}}{4} & \\
0 & -\frac{k_{2} L}{2} & 0 & 0 & 0 & -k_{6}+\frac{k_{2} L^{2}}{4}
\end{array}\right]
\end{aligned}
$$

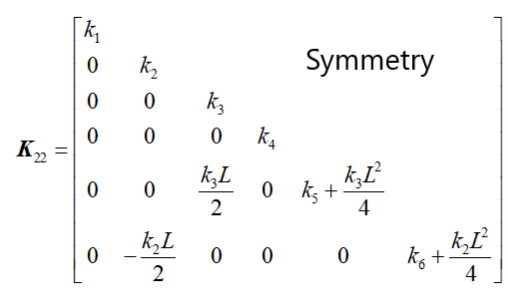

In the formula: $\mathrm{k}_{\mathrm{i}}(\mathrm{i}=1,2, \ldots, 6)$ is the six input parameters of the BUSH unit; $\mathrm{L}$ is the unit length. By consulting the literature [10], it can be seen that the stiffness matrix of the BUSH unit and the shear beam unit have the same form, and there are coupling stiffness terms of vertical displacement and rotation angle. Therefore, compared with the six-degree-of-freedom scalar spring-damping system, the BUSH unit can be more accurately equivalent to the elastic connection in the actual engineering structure.

\section{Consider natural frequencies of structures with elastic connections}

As shown in Fig. 1, it is assumed that the overall structure is composed of two parts, $\mathrm{P}$ and $\mathrm{Q}$, and $\mathrm{P}$ and $\mathrm{Q}$ are connected through $n$ elastic connections $\left(p_{i} \sim q_{i}, i=\right.$ $1,2, \ldots, n)$ of the same specification.

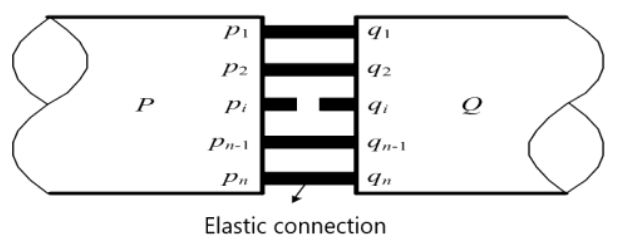

Fig. 1. Schematic chart of overall structure

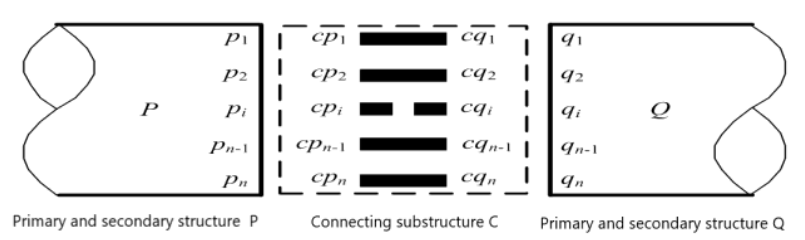

Fig. 2. Schematic chart of substructure partition

In order to improve the calculation efficiency, this study uses FIMSM to calculate the natural frequency of the structure considering elastic connection shown in Fig. $1^{[11]}$. The basic steps of FIMSM are: 1) according to the structural characteristics and analysis requirements, the overall structure is divided into sub-structures with fixed interfaces; 2) extract the modal information of each substructure based on FEM, and calculate the hypothetical mode set that each substructure participates in modal synthesis, which means the low-order retained main mode and the constrained mode; 3 ) according to the coordinated conditions of the interface displacement, the modal synthesis is performed by using the hypothesized mode set of each substructure, and the vibration equation with reduced degrees of freedom of the overall structure is established, and further calculation and analysis are carried out.

For the overall structure shown in Fig. 1, if the effect of elastic connection is ignored, it can be divided into two substructures $\mathrm{P}$ and $\mathrm{Q}$ for processing. The corresponding interface node sets $\mathrm{P}_{\mathrm{v}}$ and $\mathrm{Q}_{\mathrm{v}}$ are:

$$
P_{v}=\left\{p_{i} \mid i=1,2, \cdots, n\right\} ; Q_{v}=\left\{q_{i} \mid i=1,2, \cdots, n\right\}
$$

Literature [11] pointed out that the interfacial displacement coordination between adjacent substructures is required by FIMSM. However, for the substructure $\mathrm{P}$ and the substructure $\mathrm{Q}$ shown in Fig. 1, since the two are elastically connected, the displacements of the corresponding interface nodes in $\mathrm{P}_{\mathrm{v}}$ and $\mathrm{Q}_{\mathrm{v}}$ are not continuous. If FIMSM is used to calculate its natural frequency directly, it will cause certain errors or even errors. At this time, if the elastic connection between the substructure $\mathrm{P}$ and the substructure $\mathrm{Q}$ is divided into a connection substructure $\mathrm{C}$ having only interface nodes, not only can it meet the division 
principle of the substructure, but it can also coordinate the interface displacement of the substructure $\mathrm{P}$ and the substructure $Q$ by connecting the substructure $C$. Therefore, this study divides the overall structure shown in Fig. 1 into P, Q, and C independent substructures for processing, as shown in Fig. 2.

After the sub-structure division is completed, the physical parameters of the main sub-structure $\lambda(\lambda=P, Q)$ are first extracted based on the FEM, that is, the mass matrix $\mathrm{M}^{\lambda}$ and the stiffness matrix $\mathrm{K}^{\lambda}$; then, according to the internal node $\mathrm{u}$ and the interface node $\mathrm{v}$, the mass matrix $M^{\lambda}$ and the stiffness matrix $M^{\lambda}$ of the main substructure $\lambda$ are divided into blocks, as shown below:

$$
\begin{aligned}
M^{\lambda} & =\left[\begin{array}{ll}
M_{u u}^{\lambda} & M_{u v}^{\lambda} \\
M_{v u}^{\lambda} & M_{v v}^{\lambda}
\end{array}\right] ; \\
K^{\lambda} & =\left[\begin{array}{ll}
K_{u u}^{\lambda} & K_{u v}^{\lambda} \\
K_{v u}^{\lambda} & K_{v v}^{\lambda}
\end{array}\right]
\end{aligned}
$$

At this time, according to the definition of the main mode and the constraint mode of the substructure, the calculation formulas of the main mode $\varphi_{n}^{\lambda}$ and the constraint mode $\varphi_{c}^{\lambda}$ of the main substructure $\lambda$ are $^{[11]}$ :

$$
\begin{gathered}
\varphi_{n}^{\lambda}=\left[\begin{array}{l}
\varphi_{u n}^{\lambda} \\
O_{v n}
\end{array}\right], \quad\left(K_{u u}^{\lambda}-\omega^{2} M_{u u}^{\lambda}\right) \varphi_{u n}^{\lambda}=0 ; \\
\varphi_{c}^{\lambda}=\left[\begin{array}{l}
\varphi_{u c}^{\lambda} \\
I_{v c}
\end{array}\right], \quad \varphi_{u c}^{\lambda}=-\left(K_{u u}^{\lambda}\right)^{-1} K_{u v}^{\lambda}
\end{gathered}
$$

In the formula: $\omega$ is the angular frequency; I is the identity matrix. Suppose the first $r-\operatorname{order}(\mathrm{r}<<\mathrm{n}$, so as to reduce the degree of freedom and improve the calculation efficiency) main mode $\varphi_{r}^{\lambda}$ is selected as the lower-order retained main mode of the substructure $\lambda$, Then the hypothetical mode set $\varphi^{\lambda}$ where the substructure $\lambda$ participates in modal synthesis can be written as:

$$
\varphi^{\lambda}=\left[\begin{array}{ll}
\varphi_{r}^{\lambda} & \varphi_{c}^{\lambda}
\end{array}\right]=\left[\begin{array}{ll}
\varphi_{u r}^{\lambda} & \varphi_{u c}^{\lambda} \\
0_{v r} & I_{v c}
\end{array}\right]
$$

For the elastic connection shown in FIG. 1, due to its small mass, the influence on the vibration characteristics of the overall structure can be ignored ${ }^{[8,9,12]}$. As for the stiffness of the elastic connection, this study uses the BUSH unit to equivalent it. Therefore, the mass matrix $\mathrm{M}^{\mathrm{C}}$ and the stiffness matrix $\mathrm{K}^{\mathrm{C}}$ of the connection substructure $\mathrm{C}$ can be written as:

$$
\mathrm{M}^{\mathrm{C}}=0 ; \mathrm{K}^{\mathrm{C}}=\operatorname{diag}\left(\mathrm{K}_{\mathrm{b}}, \ldots, \mathrm{K}_{\mathrm{n}}\right)
$$

Similarly, according to equation (5), the hypothetical mode set $\varphi^{C}$ connecting the substructure $\mathrm{C}$ to participate in modal synthesis can be obtained as:

$$
\varphi^{C}=\varphi_{c}^{C}=I
$$

In the formula: $\varphi \mathrm{C}_{\mathrm{c}}$ is the constraint mode of the substructure $C$. After obtaining the mass matrix, stiffness matrix of each substructure and the set of hypothetical modes involved in modal synthesis, the mass matrix $\mathrm{M}$, stiffness matrix $\mathrm{K}$, and modal matrix $\varphi$ of the overall structure can be written as:

$$
\begin{gathered}
M=\left[\begin{array}{ccc}
M^{P} & 0 & 0 \\
0 & M^{C} & 0 \\
0 & 0 & M^{Q}
\end{array}\right]=\left[\begin{array}{ccc}
M^{P} & 0 & 0 \\
0 & 0 & 0 \\
0 & 0 & M^{Q}
\end{array}\right] ; \\
K=\left[\begin{array}{ccc}
K^{P} & 0 & 0 \\
0 & K^{C} & 0 \\
0 & 0 & K^{Q}
\end{array}\right] ; \varphi=\left[\begin{array}{ccc}
\varphi^{P} & 0 & 0 \\
0 & \varphi^{C} & 0 \\
0 & 0 & \varphi^{Q}
\end{array}\right]
\end{gathered}
$$

Let the displacement column vector of the overall structure be $\mathrm{X}$, then:

$$
X=\left[\left(X_{u}^{P}\right)^{T}\left(X_{v}^{P}\right)^{T}\left(X_{v}^{C P}\right)^{T}\left[X_{v}^{C Q}\right]^{T}\left(X_{u}^{Q}\right)^{T}\left(X_{v}^{Q}\right)^{T}\right]^{T}
$$

In the formula: $\mathrm{X} \lambda \mathrm{u}$ and $\mathrm{X} \lambda \mathrm{v}$ are displacement vector of internal node and interface node of main substructure $\lambda ; \mathrm{X} \mathrm{C} \lambda \mathrm{v}$ is the displacement vector of interface node connecting substructure $\mathrm{C}$ and main substructure $\lambda$. Let the modal coordinate of the overall structure modal matrix $\varphi$ be $\mathrm{Y}$, then:

$$
X=\varphi Y ; Y=\left[\left(Y_{r}^{P}\right)^{T}\left(Y_{c}^{P}\right)^{T}\left(Y_{c}^{C P}\right)^{T}\left(Y_{c}^{C Q}\right)^{T}\left(Y_{r}^{Q}\right)^{T}\left(Y_{c}^{Q}\right)^{T}\right]^{T}
$$

In the formula: $\mathrm{Y}_{\mathrm{r}}^{\lambda}$ and $\mathrm{Y}_{\mathrm{c}}^{\lambda}$ are the mode coordinates of the low-order retained main mode and the constrained mode of the main substructure ${ }^{\lambda}$, respectively; $\mathrm{Y}_{\mathrm{c}}^{\mathrm{C} \lambda}$ is the mode coordinates of the constrained mode connected to the substructure $\mathrm{C}$. At this time, it can be obtained that the modal mass matrix $M_{Y}$ and the modal stiffness matrix $\mathrm{K}_{\mathrm{Y}}$ whose overall structure corresponds to the modal coordinate $Y$ are:

$$
\mathrm{M}_{\mathrm{Y}}=\varphi^{\mathrm{T}} \mathrm{M} \varphi ; \quad \mathrm{K}_{\mathrm{Y}}=\varphi^{\mathrm{T}} \mathrm{K} \varphi
$$

The coordination conditions for introducing interface displacements are:

$$
X_{v}^{C P}=L_{P} X_{v}^{p} ; X_{v}^{C Q}=L_{Q} X_{v}^{Q}
$$

In the formula: $\mathrm{L}_{P}$ and $\mathrm{L}_{\mathrm{Q}}$ are coordinate rotation transformation matrices. Sorting formulas (10) to (12) and formula (13) gives:

$$
Y_{c}^{C P}=L_{P} Y_{c}^{P} ; Y_{c}^{C Q}=L_{Q} Y_{c}^{Q}
$$

An independent transformation matrix $S$ is introduced to independently transform $\mathrm{Y}$. The transformation process is as follows: 


$$
\begin{gathered}
Y=S Z=\left[\begin{array}{cccc}
I & 0 & 0 & 0 \\
0 & I & 0 & 0 \\
0 & L_{P} & 0 & 0 \\
0 & 0 & 0 & L_{Q} \\
0 & 0 & I & 0 \\
0 & 0 & 0 & I
\end{array}\right]\left[\begin{array}{c}
Y_{r}{ }^{P} \\
Y_{c}{ }^{P} \\
Y_{r}{ }^{Q} \\
Y_{c}{ }^{Q}
\end{array}\right] ; \\
S=\left[\begin{array}{cccc}
I & 0 & 0 & 0 \\
0 & I & 0 & 0 \\
0 & L_{P} & 0 & 0 \\
0 & 0 & 0 & L_{Q} \\
0 & 0 & I & 0 \\
0 & 0 & 0 & I
\end{array}\right] ; \quad Z=\left[\begin{array}{c}
Y_{r}{ }^{P} \\
Y_{r}{ }^{P} \\
Y_{r}{ }^{2} \\
Y_{c}{ }^{Q}
\end{array}\right]
\end{gathered}
$$

In the formula: $\mathrm{Z}$ is the independent modal coordinate of the overall structure. At this time, it can be obtained that the modal mass matrix $\mathrm{M}_{\mathrm{Z}}$ and the modal stiffness matrix $\mathrm{K}_{\mathrm{Z}}$ whose overall structure corresponds to the independent modal coordinate $\mathrm{Z}$ are:

$\mathrm{M}_{\mathrm{Z}}=\mathrm{S}^{\mathrm{T}} \mathrm{M}_{\mathrm{Y}} \mathrm{S}=\mathrm{S}^{\mathrm{T}} \varphi^{\mathrm{T}} \mathrm{M} \varphi \mathrm{S} ; \quad \mathrm{K}_{\mathrm{Z}}=\mathrm{S}^{\mathrm{T}} \mathrm{K}_{\mathrm{Y}} \mathrm{S}=\mathrm{S}^{\mathrm{T}} \varphi^{\mathrm{T}} \mathrm{K} \varphi \mathrm{S}$ (16)

Therefore, the undamped free vibration equation of the reduced overall degrees of freedom of the overall structure shown in Fig. 1 based on FIMSM is:

$$
M_{Z} \ddot{Z}+K_{Z} Z=0
$$

The modal matrix of the vibration equation shown in formula (17) is $\Phi$, and its calculation formula and expression are:

$$
\left(\mathrm{K}_{\mathrm{Z}}-\omega^{2} \mathrm{M}_{\mathrm{Z}}\right) \Phi=0 ; \Phi=\left[\Phi_{1}, \Phi_{2}, \ldots, \Phi_{\mathrm{i}}\right]
$$

At this time, according to the Rayleigh quotient formula, the $\mathrm{i}$-th angular frequency $\omega \mathrm{i}$ of the overall structure shown in Fig. 1 is:

$$
\omega_{i}^{2}=\frac{\phi_{i}^{T} K_{Z} \phi_{i}}{\phi_{i}^{T} M_{Z} \phi_{i}}=\frac{\phi_{i}^{T} S^{T} \varphi^{T} K \varphi S \phi_{i}}{\phi_{i}^{T} S^{T} \varphi^{T} M \varphi S \phi_{i}}
$$

The above process is to use the elastic connection in the equivalent structure of the BUSH unit and express the natural frequency of the structure considering the elastic connection based on the FIMSM derivation. Because FIMSM doesn't consider the influence of the higher-order main modes of each main substructure, the calculation efficiency can be greatly improved.

\section{Study Verification}

In order to verify the correctness of the above method, an example structure with elastic connection is designed as shown in Fig. 3. Under the premise of using the equivalent elastic connection of the BUSH unit, FIMSM and FEM are used in the first 30 steps of the structure. The frequency is calculated and the calculation results are compared.

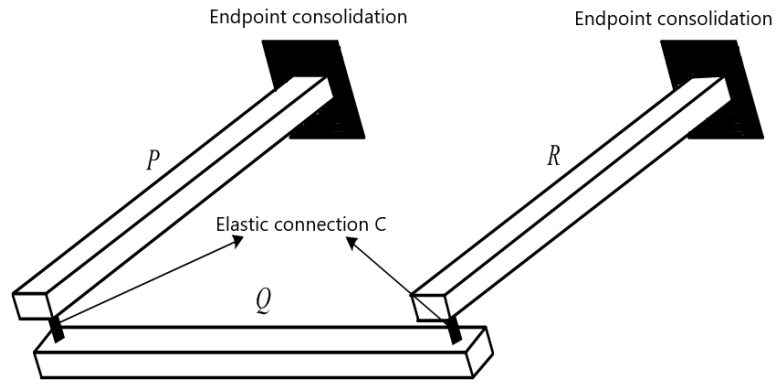

Fig. 3. Schemaric structure of example structure

In Fig. 3, $\mathrm{P}, \mathrm{Q}$ and $\mathrm{R}$ are three long beams with the same rectangular cross-section. The section size and beam length are $0.04 \mathrm{~m} \times 0.02 \mathrm{~m}$ and $0.5 \mathrm{~m}$, respectively. The elements are divided by using shear beam elements. See Table 1 for element attributes; $\mathrm{C}$ is 2 identical elastic connections, which are equivalent using BUSH units. See Table 2 for the unit properties.

Table 1. Element attributes of the shear beam.

\begin{tabular}{|c|c|c|c|c|}
\hline $\begin{array}{c}\text { Substruct } \\
\text { ure }\end{array}$ & $\begin{array}{c}\text { Elasticity } \\
\text { modulus/ } \\
\mathrm{Pa}\end{array}$ & $\begin{array}{c}\text { Poisson's } \\
\text { ratio }\end{array}$ & $\begin{array}{c}\text { Density/ } \\
\mathrm{kg} / \mathrm{m}^{3}\end{array}$ & $\begin{array}{c}\text { Length/ } \\
\mathrm{m}\end{array}$ \\
\hline $\begin{array}{c}P 、 Q \\
\text { and } R\end{array}$ & $7 \times 10^{10}$ & 0.3 & 2700 & 0.05 \\
\hline
\end{tabular}

Table 2. Element attributes of BUSH

\begin{tabular}{|c|c|c|c|c|c|c|c|}
\hline $\begin{array}{c}\text { Subs } \\
\text { truct } \\
\text { ure }\end{array}$ & $\begin{array}{c}k_{1} / \mathrm{N} \\
/ \mathrm{m}\end{array}$ & $\begin{array}{c}k_{2} / \mathrm{N} \\
/ \mathrm{m}\end{array}$ & $\begin{array}{c}k_{3} / \mathrm{N} \\
/ \mathrm{m}\end{array}$ & $\begin{array}{c}k_{4} / \mathrm{N} \\
\cdot \mathrm{m} / \mathrm{r} \\
\mathrm{ad}\end{array}$ & $\begin{array}{c}k_{5} / \mathrm{N} \\
\cdot \mathrm{m} / \mathrm{r} \\
\mathrm{ad}\end{array}$ & $\begin{array}{c}k_{6} / \mathrm{N} \\
\cdot \mathrm{m} / \mathrm{r} \\
\mathrm{ad}\end{array}$ & $L / \mathrm{m}$ \\
\hline$C$ & $\begin{array}{c}2.1 \times \\
10^{8}\end{array}$ & $\begin{array}{c}3.8 \times \\
10^{6}\end{array}$ & $\begin{array}{c}3.8 \times \\
10^{6}\end{array}$ & 650 & 850 & 850 & 0.05 \\
\hline
\end{tabular}

When using FIMSM calculations, the first 10 steps of the main substructures $\mathrm{P}, \mathrm{Q}$, and $\mathrm{R}$ are retained as loworder main modes to participate in modal synthesis. When using FEM for calculations, all modes of the overall structure are selected. The calculation results of the two methods are shown in Table 3.

Table 3. Calculating results from intrinsic frequencies

\begin{tabular}{|c|c|c|}
\hline Order numbe & FIMSM/Hz & FEM/Hz \\
\hline 1 & 37.033 & 37.033 \\
\hline 2 & 58.281 & 58.281 \\
\hline 3 & 75.885 & 75.885 \\
\hline 4 & 171.817 & 171.815 \\
\hline 5 & 243.044 & 243.041 \\
\hline 6 & 338.479 & 338.464 \\
\hline 7 & 383.041 & 383.006 \\
\hline 8 & 458.397 & 458.351 \\
\hline 9 & 462.078 & 462.039 \\
\hline 10 & 587.736 & 587.249 \\
\hline 11 & 761.824 & 761.512 \\
\hline 12 & 835.057 & 834.904 \\
\hline 13 & 940.449 & 939.298 \\
\hline 14 & 966.589 & 965.541 \\
\hline 15 & 1026.760 & 1026.619 \\
\hline
\end{tabular}




\begin{tabular}{|l|l|l|}
\hline 16 & 1183.474 & 1182.542 \\
\hline 17 & 1464.751 & 1462.354 \\
\hline 18 & 1788.334 & 1787.330 \\
\hline 19 & 1947.586 & 1940.273 \\
\hline 20 & 1953.285 & 1945.861 \\
\hline 21 & 2050.529 & 2048.535 \\
\hline 22 & 2230.566 & 2226.550 \\
\hline 23 & 2318.983 & 2306.868 \\
\hline 24 & 2338.818 & 2327.914 \\
\hline 25 & 2357.539 & 2330.467 \\
\hline 26 & 2592.201 & 2577.911 \\
\hline 27 & 2598.795 & 2587.836 \\
\hline 28 & 3063.926 & 3057.575 \\
\hline 29 & 3397.919 & 3394.079 \\
\hline 30 & 3654.106 & 3607.072 \\
\hline
\end{tabular}

As can be seen from Table 3, compared with the traditional FEM, the relative calculation error of the FIMSM for the first 30 natural frequencies of the structure shown in Figure 3 does not exceed $\pm 1.31 \%$. In summary, using the equivalent elastic connection of the BUSH unit, FIMSM can accurately and efficiently calculate the natural frequency of the structure considering the elastic connection.

\section{5 conlusion}

In this study, the equivalent elastic connection of BUSH elements in MSC. Nastran software was used. Based on the FIMSM, the natural frequency of the structure considering the elastic connection was theoretically derived, and the correctness of the method was verified by numerical simulation. The results show that using the equivalent elastic connection of the BUSH unit, FIMSM can accurately and efficiently predict the vibration environment with elastic connection structure. The conclusions of this study have certain theoretical research and engineering application value.

\section{References}

1. Deyuan Y, Qizheng W. Principle and Application of Statistical Energy Analysis [M]. Beijing: Beijing Institute of Technology Press, 1995

2. Dong W, Wei L. Research on Application of Aircraft Dynamic Environment Predictive Technology [J]. Modern Defence Technology, 2000, 28 (2): 28-33

3. Hongjie Y, Jun Y, Zhiqiang L. Vibration, shock environment and test [M]. Beijing: Beihang University Press, 2017

4. Liemin C. Spacecraft structure and mechanism [M]. Beijing: China Science and Technology Press, 2005

5. Xiaohan L, Bingyuan Y. Linker Structure in Structural Vibration Analysis [J] .Journal of Astronautics, 1987,8 (1): 10-18

6. Wei W, Yujin H, Ling L. Comprehensive Method and Implementation of Substructure Frequency
Response Considering Connection Characteristics [J]. China Mechanical Engineering, 2013, 24 (10): 1385-1389

7. Yuanjie Z. Bush finite element principle and its application in spacecraft structure modeling [J]. Spacecraft Engineering, 2010, 19 (1): 99-105

8. Wei W. Research and Implementation of Substructure Hybrid Modeling Based on Frequency Response Function [D]. Wuhan: Huazhong University of Science and Technology, 2012

9. Jiangpan C. Research on micro-vibration characteristics of spacecraft moving parts based on uncertain installation interface [D]. Beijing: Beijing University of Aeronautics and Astronautics, 2017

10. Yufeng X,Min L. Principles and Methods of Computational Solid Mechanics [M]. Beijing: Beijing University of Aeronautics and Astronautics Press, 2011

11. Yongyan W. Theory and Application of Dynamic Substructure Method [M]. Beijing: Science Press, 1999

12. LIU W, EWINS DJ. Substructure synthesis via elastic media[J]. Journal of Sound and Vibration, 2002,257(2):361-379 